\title{
REABILITAÇÃO RESPIRATÓRIA EM IDOSOS, EM CONTEXTO DE CUIDADOS AGUdOS: REVISÃo SISTEMÁTICA DA LITERATURA
}

Respiratory Rehabilitation in the Elderly, in the Context of Acute Care: Systematic Review of LITERATURE

\section{Rehabilitación Respiratoria en AnCianos, en el Contexto de Atención Aguda: Revisión Sistemática de LA LITERATURA}

DOI 10.33194/rper.2022.186

Data de Receção: 2021-09-20 Data de Aceitação: 2021-12-21 Data de publicação on-line: 2022-01-16

\author{
Joana Alves ${ }^{1}$; Eugénia Grilo (i) ${ }^{2}$ \\ ${ }^{1}$ Centro Hospitalar e Universitário do Algarve, Faro, Portugal; \\ ${ }^{2}$ Instituto Politécnico de Castelo Branco Escola Superior de Saúde Dr. Lopes Dias, Portugal
}

\author{
Autor correspondente: Joana Alves, joana.claudiaa@hotmail.com
}

\section{RESUMO}

Introdução: As alterações multiorgânicas presentes no processo de envelhecimento associadas a uma situação de doença podem intensificar o declínio funcional e provocar uma maior fragilidade cardiorrespiratória.

Objetivo: Identificar as intervenções suscetíveis de melhorarem o desempenho respiratório e a capacidade funcional nas pessoas idosas em contexto de agudização.

Metodologia: Revisão sistemática da literatura segundo o protocolo Joanna Briggs Institute através de pesquisa nas bases de dados de artigos científicos pela plataforma EBSCOhost, sendo incluídos artigos publicados de 2015 a 2021, através da utilização dos Medical Subject Heading.

Resultados: Após a pesquisa realizada foram identificados 144 artigos, dos quais sete respondiam aos critérios de inclusão definidos: quatro estudos clínicos randomizados, dois estudos prospetivos quase experimentais e um estudo de corte transversal.

Conclusão: De acordo com os autores dos estudos incluídos nesta revisão da literatura, as intervenções de reabilitação respiratória, como a técnica de controlo da respiração e o uso de dispositivos para treino dos músculos respiratórios permitiram a redução da fadiga, o aumento da tolerância ao esforço e a melhoria dos volumes e das capacidades pulmonares, com resultados favoráveis no desempenho funcional.

DESCRITORES: terapia respiratória, exercícios respiratórios, idoso

\section{ABSTRACT}

Introduction: The multiorgan changes present in the aging process associated with a disease situation can intensify the functional decline and cause greater cardiorespiratory frailty.

Objectives: Identify interventions likely to improve respiratory performance and functional capacity in elderly people in an acute setting.

Methodology: Systematic literature review according to the Joanna Briggs Institute protocol through research in scientific articles databases by the EBSCOhost platform, including articles published from 2015 to 2021, using the Medical Subject Heading.

Results: After the search, 144 articles were found, of which seven responded to the defined inclusion criteria. Four randomized clinical trials, two prospective quasi-experimental studies and one cross-sectional study were identified.

Conclusion: According to the authors of the studies included in the literature review, respiratory rehabilitation interventions, such as the breathing control technique, the use of devices to train the respiratory muscles, allowed for a reduction in fatigue, an increase in effort tolerance, the improvement of lung volumes and capacities, with favorable results in functional performance. 
DESCRIPTORS: Respiratory therapy, Breathing exercises, Aged

\section{RESUMEN}

Introducción: Los cambios multiorgánicos presentes en el proceso de envejecimiento asociados a una situación patológica pueden intensificar el deterioro funcional y provocar una mayor fragilidad cardiorrespiratoria.

Objetivos: Identificar intervenciones que puedan mejorar el rendimiento respiratorio y la capacidad funcional en personas mayores en un entorno agudo.

Metodología: Revisión sistemática de la literatura según el protocolo del Instituto Joanna Briggs a través de la investigación en bases de datos de artículos científicos por la plataforma EBSCOhost, incluyendo artículos publicados desde 2015 hasta 2021, utilizando el Medical Subject Heading.

Resultados: Tras la búsqueda se encontraron 144 artículos, de los cuales siete respondieron a los criterios de inclusión definidos. Se identificaron cuatro ensayos clínicos aleatorios, dos estudios cuasiexperimentales prospectivos y un estudio transversal.

Conclusión: Según los autores de los estudios incluidos en la revisión de la literatura, las intervenciones de rehabilitación respiratoria, como la técnica de control de la respiración, el uso de dispositivos para entrenar la musculatura respiratoria, permitieron una reducción de la fatiga, un aumento de la tolerancia al esfuerzo, la mejora de la volúmenes y capacidades pulmonares, con resultados favorables en el desempeño funcional.

DESCRIPTORES: Terapia respiratoria, Ejercicios respiratorios, Anciano

\section{INTRODUÇÃO}

As alterações atribuídas ao processo de envelhecimento, podem levar a uma fragilidade multiorgânica e em termos cardiopulmonares, estas alterações podem ser verificadas na diminuição da distensibilidade da parede torácica, do reflexo de tosse, na redução da atividade ciliar, no aumento do risco de infeção pulmonar, na diminuição da capacidade vital e na consequente redução da capacidade de desempenho das atividades da vida diária ${ }^{(1)}$.

Estas alterações, normais no processo de envelhecimento, podem ser intensificadas por uma situação de doença, causando desta forma um declínio funcional, de difícil resolução se não for devidamente assistido ${ }^{(1)}$.

A reabilitação, como especialidade multidisciplinar, permite recuperar a máxima independência e funcionalidade das pessoas de modo a diminuir o impacto das incapacidades instaladas, devido a patologias agudas ou crónicas, com vista à reintegração social ${ }^{(2)}$.

A reabilitação respiratória procura assegurar que as alterações fisiológicas resultantes dos desequilíbrios da relação ventilação/perfusão sejam resolvidas para que não existam repercussões funcionais ${ }^{(3)}$.

Os principais objetivos da reabilitação respiratória são reduzir os sintomas e melhorar a qualidade de vida das pessoas e em doentes com doença pulmonar obstrutiva crónica, incluem o treino de exercício, o aconselhamento nutricional e a educação ${ }^{(4)}$.

$\mathrm{Na}$ reabilitação respiratória são usados exercícios com técnicas posturais, manuais e cinéticas dos segmentos torácicos e abdominais ${ }^{(5)}$, que permitem prevenir e corrigir posições viciosas, promover o relaxamento e o controlo ventilatório, com a redução da tensão psíquica e muscular, assegurar a permeabilidade das vias aéreas e permitir uma reeducação para o esforço com exercícios de conservação de energia na realização de atividades da vida diária ${ }^{(3)}$. Estes exercícios atuam no processo mecânico da ventilação, isto é, a partir da ventilação externa é possível melhorar a ventilação alveolar ${ }^{(3)}$.

Os programas de reabilitação respiratória permitem a melhoria da capacidade de exercício e da capacidade funcional, com aumento da participação em atividades físicas e sociais e consequente aumento da qualidade de vida das pessoas ${ }^{(6)}$.

Contudo, cada programa de reabilitação respiratória deve ser adaptado individualmente, tendo em conta a patologia em questão, a idade da pessoa, a capacidade de aprendizagem e motivação, história clínica, familiar e social, as comorbilidades existentes, os objetivos e resultados esperados ${ }^{(3,5)}$, pois só assim é possível atingir os níveis máximos de independência e de funcionalidade ${ }^{(6)}$.

Definiu-se como objetivo desta revisão sistemática da literatura identificar intervenções de reabilitação respiratória suscetíveis de melhorarem o desempenho respiratório e a capacidade funcional nas pessoas idosas em contexto de agudização, tendo em conta a falta de revisões sistemáticas da temática referida. 


\section{METODOLOGIA}

Revisão sistemática da literatura tendo por base as instruções do Joanna Briggs Institute $(\mathrm{JBI})^{(7,8)}$.

Foi realizada uma primeira pesquisa acerca da temática na plataforma de EBSCOhost, tendo em conta a disponibilidade de texto integral com publicação nos últimos cinco anos. 0 objetivo da primeira pesquisa foi identificar as intervenções suscetíveis de melhorarem o desempenho respiratório e a capacidade funcional nas pessoas idosas.

Posteriormente e com base na estratégia PICO - População, Intervenção, Comparação e Resultados (outcome), foi formulada a questão “quais os benefícios $(0)$ da reabilitação respiratória (I), realizada por profissionais de saúde, em pessoas idosas (P), em contexto de agudização de doença (C)?”, a qual norteou a segunda pesquisa (Quadro 1), no sentido de identificar os benefícios concretos para as pessoas idosas resultantes dessas mesmas intervenções.

\section{Quadro 1 - Estratégia PICO}

\begin{tabular}{|c|c|}
\hline \multicolumn{2}{|c|}{ PICO } \\
\hline Pessoa / População & Adultos e Idosos \\
\hline Intervenção & Reabilitação respiratória \\
\hline Contexto & Cuidados agudos \\
\hline Outcome - Resultado & Benefícios \\
\hline
\end{tabular}

A pesquisa foi realizada entre os dias 1 de janeiro de 2021 e 6 de maio de 2021 e recorreu-se aos descritores Medical Subject Heading (MeSH), organizados com o operador booleano AND e NOT, com a seguinte sequência de pesquisa:

respiratory therapy AND breathing exercises [698 artigos];

respiratory therapy AND breathing exercises AND aged [158 artigos];

respiratory therapy AND breathing exercises AND aged NOT children [144 artigos].

$\mathrm{Na}$ pesquisa realizada na plataforma EBSCOhost com a utilização dos descritores mencionados, foram identificados inicialmente 144 artigos [MEDLINE Complete (115), CINAHL Complete (21), SPORTDiscus with full test (2), Complementary Index (1), Academic Search Complete (1)].

Posteriormente os artigos foram selecionados de acordo com os seguintes critérios de inclusão e exclusão demonstrados no quadro 2.

Quadro 2 - Critérios de inclusão e exclusão

\begin{tabular}{|c|c|}
\hline Critérios de inclusão & Critérios de exclusão \\
\hline $\begin{array}{l}\text { - Intervenções de reabilitação respiratória dirigidas a pessoas adultas e } \\
\text { idosas com patologia respiratória ou outras comorbilidades que afetem a } \\
\text { função respiratória } \\
\text { - Intervenções realizadas em cuidados de ambulatório } \\
\text { - Estudos quantitativos } \\
\text { - Limite temporal: } 2015-2021 \\
\text { - Artigo redigido em língua portuguesa ou inglesa } \\
\text { - Revistas analisadas pelos pares } \\
\text { - Texto integral disponível }\end{array}$ & $\begin{array}{l}\text { - Intervenções realizadas em } \\
\text { cuidados intensivos } \\
\text { - Intervenções realizadas em } \\
\text { doentes com ventilação assistida } \\
\text { - Intervenções realizadas em lares } \\
\text { - Pacientes submetidos a cirurgia } \\
\text { cardiotorácica }\end{array}$ \\
\hline
\end{tabular}

Dos 144 artigos identificados, foram posteriormente selecionados os artigos disponíveis em texto integral (84) e analisados por pares (82) dentro do limite temporal 2015-2021, restando 31 artigos. Destes, foi eliminado 1 artigo escrito em chinês, 1 artigo escrito em espanhol e outro por se encontrar repetido, restando 28 artigos. Três artigos foram excluídos por abordarem doentes com ventilação mecânica assistida e 3 de doentes sujeitos a cirurgia cardiotorácica. Dois artigos foram excluídos por serem revisões integrativas, ficando 20 artigos. Após a leitura destes artigos, 13 foram excluídos por não abordarem a temática de acordo com os critérios pré-estabelecidos, restando 7 artigos que cumpriam os critérios de inclusão. A figura 1 descreve o processo de seleção dos artigos, com recurso ao PRISMA Flow Diagram. 
Após a seleção dos artigos e para determinar a confiabilidade dos mesmos, foi realizada a avaliação metodológica dos estudos e determinado o seu nível de evidência segundo as normas de $\mathrm{JBI}^{(7)}$, aplicando as Checklists Critical Appraisal Tools, com o objetivo de avaliar a qualidade metodológica do estudo e determinar a sua confiabilidade, relevância e avaliar a evidência dos resultados dos artigos publicados (JBI, 2020) igualmente com a utilização do documento JBI Levels of Evidence ${ }^{(9)}$. Com base no documento JBI Grades of Recommendation ${ }^{(10)}$ determinou-se o grau de recomendação dos 7 artigos. As tabelas 1 e 2 apresentam, os níveis de evidência e grau de recomendação de todos os artigos incluídos.

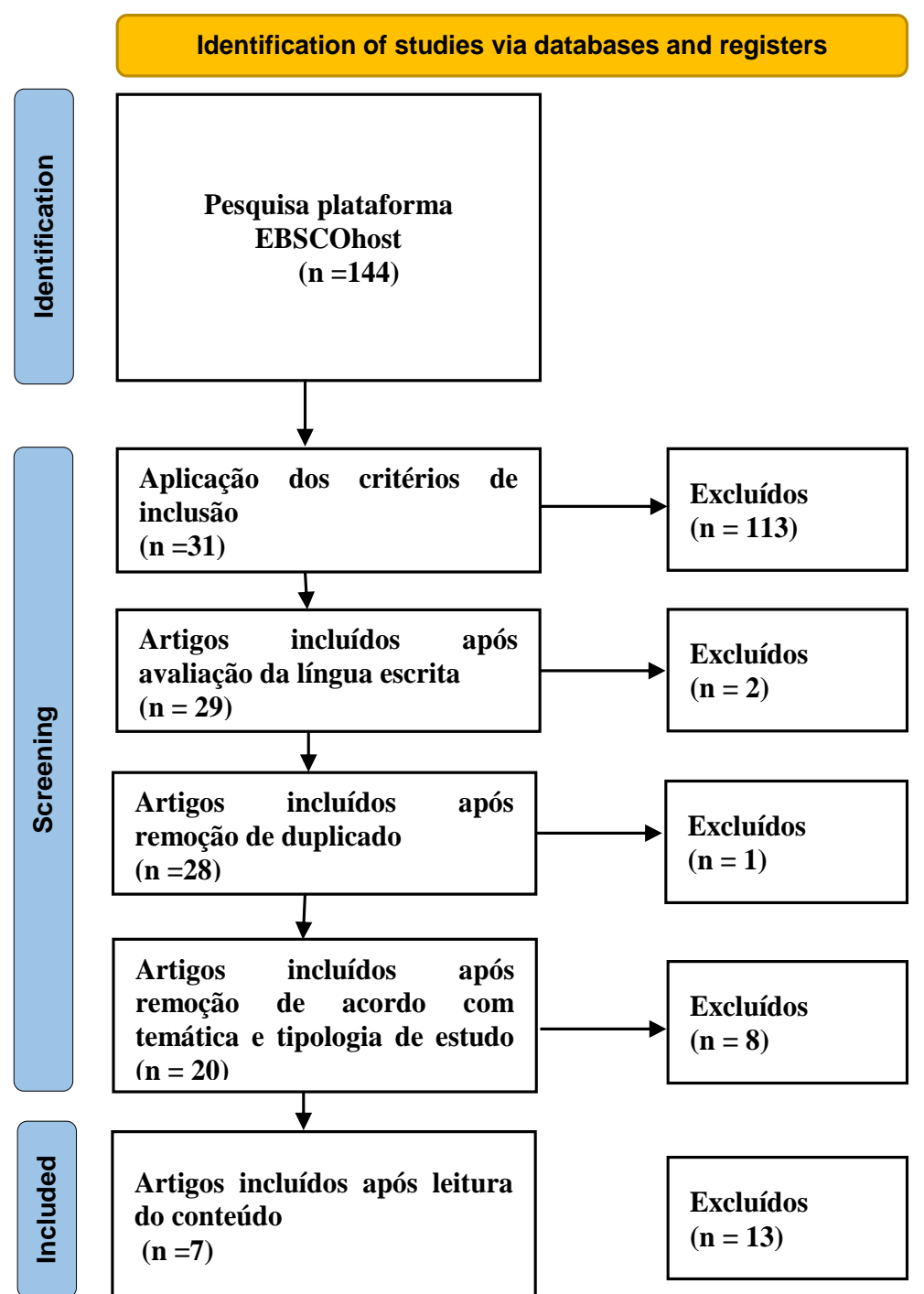

Figura 1 - Metodologia de pesquisa Prisma Flow Diagram

Tabela 1 - Classificação dos estudos segundo o nível de evidência e recomendação

\begin{tabular}{|c|l|l|c|}
\hline Estudo & Ano & \multicolumn{1}{|c|}{ Nível evidência } & Grau de recomendação \\
\hline 11 & 2015 & 1.c - Randomized controlled trial & strong \\
\hline 12 & 2020 & 1.c - Randomized controlled trial & strong \\
\hline 13 & 2020 & 4. b - Cross-sectional study & strong \\
\hline 14 & 2018 & 1.c - Randomized controlled trial & strong \\
\hline 15 & 2020 & 2.c - Quasi-experimental prospectively controlled study & strong \\
\hline 16 & 2018 & 2.c - Quasi-experimental prospectively controlled study & strong \\
\hline 17 & 2021 & 1.c - Randomized controlled trial & \\
\hline
\end{tabular}


Tabela 2 - Resultado da aplicação da Checklists Critical Appraisal Tools

\begin{tabular}{|c|c|c|c|c|c|c|c|c|c|c|c|c|c|c|}
\hline Estudo & Q1 & Q2 & Q3 & Q4 & Q5 & Q6 & Q7 & Q8 & Q9 & Q10 & Q11 & Q12 & Q13 & X\% \\
\hline 11 & $\mathrm{Y}$ & $\mathrm{Y}$ & $\mathrm{Y}$ & $\mathrm{U}$ & $\mathrm{N}$ & $\mathrm{U}$ & $\mathrm{Y}$ & $\mathrm{Y}$ & $\mathrm{Y}$ & $\mathrm{Y}$ & $\mathrm{Y}$ & $\mathrm{Y}$ & $\mathrm{Y}$ & 90,9 \\
\hline 12 & $\mathrm{Y}$ & $\mathrm{Y}$ & $\mathrm{Y}$ & $\mathrm{Y}$ & $\mathrm{N}$ & $\mathrm{U}$ & $\mathrm{Y}$ & $\mathrm{Y}$ & $\mathrm{Y}$ & $\mathrm{Y}$ & $\mathrm{Y}$ & $\mathrm{Y}$ & $\mathrm{Y}$ & 91,6 \\
\hline 13 & $\mathrm{Y}$ & $\mathrm{Y}$ & $\mathrm{Y}$ & $\mathrm{Y}$ & $\mathrm{Y}$ & $\mathrm{Y}$ & $\mathrm{Y}$ & $\mathrm{Y}$ & - & - & - & - & - & 100 \\
\hline 14 & $\mathrm{Y}$ & $\mathrm{Y}$ & $\mathrm{Y}$ & $\mathrm{Y}$ & $\mathrm{U}$ & $\mathrm{Y}$ & $\mathrm{Y}$ & $\mathrm{Y}$ & $\mathrm{Y}$ & $\mathrm{Y}$ & $\mathrm{Y}$ & $\mathrm{Y}$ & $\mathrm{Y}$ & 100 \\
\hline 15 & $\mathrm{Y}$ & $\mathrm{Y}$ & $\mathrm{Y}$ & $\mathrm{Y}$ & $\mathrm{N}$ & $\mathrm{Y}$ & $\mathrm{Y}$ & $\mathrm{Y}$ & $\mathrm{Y}$ & - & - & - & - & 88,9 \\
\hline 16 & $\mathrm{Y}$ & $\mathrm{Y}$ & $\mathrm{Y}$ & $\mathrm{N}$ & $\mathrm{Y}$ & $\mathrm{Y}$ & $\mathrm{Y}$ & $\mathrm{Y}$ & $\mathrm{Y}$ & - & - & - & - & 88,9 \\
\hline 17 & $\mathrm{Y}$ & $\mathrm{Y}$ & $\mathrm{Y}$ & $\mathrm{N}$ & $\mathrm{N}$ & $\mathrm{Y}$ & $\mathrm{Y}$ & $\mathrm{Y}$ & $\mathrm{Y}$ & $\mathrm{Y}$ & $\mathrm{Y}$ & $\mathrm{Y}$ & $\mathrm{Y}$ & 84,6 \\
\hline
\end{tabular}

Legenda: Y-Yes; N-No; U-Unclear.

Tabela 3 - Caraterização dos artigos selecionados

\begin{tabular}{|c|c|c|c|c|c|c|}
\hline $\mathbf{E}$ & Pessoa/População & $\begin{array}{l}\text { Contexto/ } \\
\text { Período }\end{array}$ & Objetivos & $\begin{array}{c}\text { Intervenções de reabilitação } \\
\text { respiratória /pulmonar }\end{array}$ & $\begin{array}{l}\text { Quem } \\
\text { interveio }\end{array}$ & $\begin{array}{l}\text { Resultados } \\
\text { Benefícios }\end{array}$ \\
\hline 11 & $\begin{array}{l}62 \text { pacientes ( } 31 \\
\text { grupo experimental, } \\
31 \text { grupo de controlo), } \\
\text { com média de idade } \\
70 \text { anos, conscientes } \\
\text { com DPOC, com } \\
\text { dispneia. }\end{array}$ & $\begin{array}{l}\text { Dois serviços de } \\
\text { internamento } \\
\text { de pneumologia } \\
\text { Taiwan } \\
\\
11 / 2011 \text { a } \\
4 / 2012\end{array}$ & \begin{tabular}{l|} 
Avaliar os efeitos de \\
exercícios de \\
reabilitação \\
respiratória na \\
dispneia, tosse, \\
tolerância ao \\
exercício em idosos \\
com DPOC, \\
hospitalizados
\end{tabular} & \begin{tabular}{|l|} 
Intervenções de reabilitação \\
respiratória com 6 componentes: \\
Consciencialização da doença; \\
técnicas de limpeza de vias \\
aéreas, técnica de respiração com \\
lábios semicerrados, exercícios de \\
membros superiores com \\
respiração profunda, treino de \\
marcha, e educação para a saúde. \\
Sessões 2 vezes por dia durante 10 \\
minutos, durante 4 dias.
\end{tabular} & $\begin{array}{l}\text { Enfermeiros e } \\
\text { médico }\end{array}$ & $\begin{array}{l}\text { Ao } 4^{\circ} \text { dia o grupo } \\
\text { experimental } \\
\text { apresentava menos } \\
\text { dispneia, menos } \\
\text { frequência de tosse e } \\
\text { maior tolerância ao } \\
\text { exercício. }\end{array}$ \\
\hline 12 & $\begin{array}{l}21 \text { pacientes entre os } \\
35 \text { e } 80 \text { anos, com } \\
\text { Acidente Vascular } \\
\text { Cerebral fraqueza dos } \\
\text { músculos } \\
\text { respiratórios, disfagia } \\
\text { ou disartria. Grupo } \\
\text { controlo } n=10 \text {, grupo } \\
\text { experimental } n=11\end{array}$ & $\begin{array}{l}\text { Hospital em } \\
\text { Taiwan } \\
\text { Abril } 2016 \text { a } \\
\text { outubro } 2018\end{array}$ & \begin{tabular}{|l|} 
Avaliar eficácia do \\
treino dos músculos \\
respiratórios em \\
relação à \\
deglutição, função \\
pulmonar, \\
desempenho \\
funcional e disartria \\
em pacientes com \\
AVC
\end{tabular} & $\begin{array}{l}\text { Treino de músculos inspiratórios e } \\
\text { expiratórios com dispositivo de } \\
\text { treino de músculos respiratórios } \\
\text { (Dofin Breathing Trainer), } \\
\text { treino postural, } \\
\text { controlo de respiração, } \\
\text { técnica de tosse. } \\
\text { Conjunto de exercícios durante } 6 \\
\text { semanas. }\end{array}$ & $\begin{array}{l}\text { Médico e } \\
\text { técnico }\end{array}$ & $\begin{array}{l}\text { O treino dos músculos } \\
\text { respiratórios } \\
\text { melhorou a } \\
\text { capacidade vital } \\
\text { forçada, o volume } \\
\text { expiratório forçado } \\
\text { por segundo e } \\
\text { melhorou a fadiga em } \\
\text { doentes com AVC. }\end{array}$ \\
\hline 13 & $\begin{array}{l}16 \text { pacientes com } \\
\text { DPCO leve a } \\
\text { moderada, com } \\
\text { dispneia leve a } \\
\text { moderada, } \\
\text { fumadores. } \\
\text { Participantes com } \\
\text { média de idades } \\
\text { superior a } 60 \text { anos. }\end{array}$ & $\begin{array}{l}\text { Departamento } \\
\text { de Fisioterapia } \\
\text { da Faculdade } \\
\text { de ciências da } \\
\text { saúde Allied. } \\
\text { Tailandia. } \\
\text { Uma avaliação }\end{array}$ & $\begin{array}{l}\text { Avaliar os efeitos da } \\
\text { respiração com os } \\
\text { lábios semicerrados } \\
\text { e diferentes } \\
\text { posturas do tronco, } \\
\text { nos volumes } \\
\text { pulmonares e } \\
\text { ventilação em } \\
\text { pacientes com } \\
\text { DPOC. }\end{array}$ & $\begin{array}{l}\text { Monitorização dos volumes } \\
\text { pulmonares e da ventilação em } \\
\text { diferentes posturas, ereta e } \\
\text { sentado com inclinação do tronco } \\
\text { a } 45^{\circ} \text { e com } 2 \text { padrões respiratórios } \\
\text { com uma respiração espontânea e } \\
\text { inspiração pelo nariz e expiração } \\
\text { pela boca com os lábios } \\
\text { semicerrados. }\end{array}$ & \begin{tabular}{|c|} 
Equipa de \\
investigadores \\
do \\
Departamento \\
de Fisioterapia \\
da Faculdade \\
de ciências da \\
saúde Allied. \\
Tailandia.
\end{tabular} & $\begin{array}{l}\text { A inspiração pelo } \\
\text { nariz e expiração pela } \\
\text { boca com lábios } \\
\text { semicerrados alterou } \\
\text { positivamente os } \\
\text { volumes pulmonares e } \\
\text { a ventilação em } \\
\text { pacientes com DPOC } \\
\text { leve a moderada. }\end{array}$ \\
\hline 14 & $\begin{array}{l}14 \text { pacientes com } \\
\text { doença de Parkinson } \\
\text { de forma leve a } \\
\text { moderada, entre os } \\
52 \text { e os } 79 \text { anos. }\end{array}$ & $\begin{array}{l}\text { Departamento } \\
\text { de fisioterapia } \\
\text { da Universidade } \\
\text { Federal de } \\
\text { Pernanbuco, } \\
\text { Brasil } \\
\\
\text { O estudo foi } \\
\text { realizado } \\
\text { durante quatro } \\
\text { dias não } \\
\text { consecutivos } \\
\text { com intervalo } \\
\text { de sete dias }\end{array}$ & \begin{tabular}{|l|} 
Avaliar os efeitos \\
das técnicas de \\
Breath Stacking e \\
inspirometria de \\
incentivo nas \\
variações de volume \\
da parede torácica \\
em pacientes com \\
doença de \\
Parkinson
\end{tabular} & $\begin{array}{l}\text { Ensino/treino sobre a realização } \\
\text { da técnica de Breath Stacking e } \\
\text { inspirometria de incentivo e } \\
\text { avaliação das pressões } \\
\text { inspiratórias e expiratórias } \\
\text { máximas, a partir do volume } \\
\text { residual e capacidade pulmonar } \\
\text { total e avaliado o volume da caixa } \\
\text { torácica, toraco abdominal e } \\
\text { abdominal. }\end{array}$ & $\begin{array}{c}\text { Equipa } \\
\text { investigadores } \\
\text { do } \\
\text { Departamento } \\
\text { de Fisioterapia } \\
\text { da Universidade } \\
\text { Federal de } \\
\text { Pernabuco, } \\
\text { Brasil. }\end{array}$ & $\begin{array}{l}\text { Após a aplicação da } \\
\text { técnica de Breath } \\
\text { Stacking e } \\
\text { inspirometria de } \\
\text { incentivo verificaram } \\
\text { um aumento do } \\
\text { volume corrente nos } \\
\text { doentes com doença } \\
\text { de Parkinson. } \\
\text { Não se verificaram } \\
\text { diferenças entre as } \\
\text { técnicas. }\end{array}$ \\
\hline
\end{tabular}




\begin{tabular}{|c|c|c|c|c|c|c|}
\hline E & Pessoa/População & $\begin{array}{l}\text { Contexto/ } \\
\text { Período }\end{array}$ & Objetivos & $\begin{array}{c}\text { Intervenções de reabilitação } \\
\text { respiratória /pulmonar }\end{array}$ & $\begin{array}{l}\text { Quem } \\
\text { interveio }\end{array}$ & $\begin{array}{l}\text { Resultados } \\
\text { Benefícios }\end{array}$ \\
\hline 15 & $\begin{array}{l}18 \text { pacientes no grupo } \\
\text { experimental e } 16 \\
\text { pacientes no grupo de } \\
\text { Controlo. } \\
\text { Participantes com } \\
\text { idade média superior } \\
\text { a } 40 \text { anos. }\end{array}$ & $\begin{array}{l}\text { Ambulatório/ } \\
\text { domicílio, } \\
\text { Taiwan } \\
12 \text { semanas }\end{array}$ & $\begin{array}{l}\text { Avaliar a eficácia do } \\
\text { treino dos músculos } \\
\text { respiratórios nos } \\
\text { resultados } \\
\text { funcionais e } \\
\text { redução da fadiga } \\
\text { em pacientes com } \\
\text { miastenia gravis. }\end{array}$ & $\begin{array}{l}\text { Treino dos músculos respiratórios } \\
\text { com Dofin } \\
\text { Breathing Trainer, (dispositivo } \\
\text { portátil de limite de pressão) foi } \\
\text { aplicado } \\
\text { para gerar força expiratória para a } \\
\text { função da tosse e } \\
\text { esforço muscular inspiratório para } \\
\text { as deficiências da ventilação } \\
\text { pulmonar }\end{array}$ & Terapeuta & $\begin{array}{l}\text { Verificaram um } \\
\text { aumento significativo } \\
\text { da capacidade vital } \\
\text { forçada, do volume } \\
\text { expiratório forçado no } \\
\text { primeiro segundo, da } \\
\text { distância de } \\
\text { caminhada de } 6 \\
\text { minutos e diminuição } \\
\text { da fadiga segundo a } \\
\text { escala } \\
\text { Multidimensional } \\
\text { Fatigue Symptom } \\
\text { Inventory-Short Form } \\
\text { (MFSI-SF) }\end{array}$ \\
\hline 17 & $\begin{array}{l}60 \text { pacientes com } \\
\text { asma entre os } 45 \text { e os } \\
60 \text { anos }\end{array}$ & $\begin{array}{l}\text { Ambulatório } \\
\text { Taiwan } \\
12 \text { semanas }\end{array}$ & $\begin{array}{l}\text { Comparar os efeitos } \\
\text { de exercícios } \\
\text { respiratórios } \\
\text { convencionais e do } \\
\text { treino dos músculos } \\
\text { inspiratórios nos } \\
\text { sintomas de doentes } \\
\text { asmáticos }\end{array}$ & $\begin{array}{l}1^{\circ} \text { grupo: } \\
\text {-Realização de exercícios de } \\
\text { alongamento dos músculos } \\
\text { torácicos } \\
\text {-Realização de inspiração nasal } \\
\text { com expiração com lábios } \\
\text { semicerrados e respiração } \\
\text { diafragmática. } \\
2^{\circ} \text { grupo: } \\
\text {-Treino dos músculos inspiratórios } \\
\text { com início de cada respiração pelo } \\
\text { volume residual até à pressão } \\
\text { inspiratória máxima, através do } \\
\text { uso de um dispositivo de treino dos } \\
\text { músculos inspiratórios. }\end{array}$ & Enfermeiros & $\begin{array}{l}\text { Não se verificaram } \\
\text { alterações na } \\
\text { capacidade vital } \\
\text { forçada e no volume } \\
\text { expiratório forçado } \\
\text { em um segundo, em } \\
\text { ambos os grupos. } \\
\text { Nos dois grupos foi } \\
\text { verificado aumento da } \\
\text { pressão expiratória } \\
\text { máxima, contudo só } \\
\text { houve aumento da } \\
\text { pressão inspiratória } \\
\text { máxima no grupo de } \\
\text { treino dos músculos } \\
\text { inspiratórios. } \\
\text { Nos dois grupos houve } \\
\text { aumento da } \\
\text { capacidade de marcha } \\
\text { durante } 6 \text { minutos. } \\
\text { Não se verificou } \\
\text { diferença na } \\
\text { saturação de oxigénio } \\
\text { ou frequência } \\
\text { cardíaca. }\end{array}$ \\
\hline
\end{tabular}

Legenda: E- Estudo

\section{RESULTADOS}

De acordo com os critérios de inclusão e exclusão pré-definidos e a partir da revisão da literatura foram selecionados sete artigos publicados entre 2015 e 2021, cujos resultados estão apresentados na tabela 3, analisados a partir do protocolo de pesquisa PICO.

Os artigos resultaram de estudos realizados em Taiwan, Brasil e Tailândia e a intervenção de reabilitação respiratória foi realizada em indivíduos adultos e idosos por vários grupos profissionais. Num artigo as intervenções foram realizadas por enfermeiros; noutro artigo as intervenções foram realizadas por terapeutas; noutro artigo as intervenções foram realizadas por fisioterapeutas; noutro artigo as intervenções foram realizadas por médicos e enfermeiros e noutro por médicos e técnicos, sem que seja clarificado qual o profissional que realizou cada uma 
das intervenções e por fim, noutros 2 artigos as intervenções foram realizadas por investigadores sem que seja especificado o grupo profissional.

No que se refere à duração das intervenções verificou-se uma grande variabilidade, dois dos estudos relatam apenas uma única sessão de intervenções e os restantes estudos relatam sessões que variaram entre 4 dias e 12 semanas.

\section{DISCUSSÃO}

A análise dos estudos selecionados permitiu identificar alguns dos benefícios concretos da reabilitação respiratória em pessoas adultas e idosas utilizando intervenções individualizadas em função do ciclo da vida das pessoas, mas também da condição de saúde das mesmas e baseadas na melhor evidência científica ${ }^{(4,18)}$.

Os artigos analisados demonstraram que a reabilitação respiratória, promove a redução dos sintomas e deste modo aumenta a capacidade de desempenho nas atividades da vida diária e nas atividades sociais com ganhos na qualidade de vida das pessoas com patologia respiratória.

A reabilitação respiratória deve ser idealmente implementada por uma equipa multidisciplinar constituída por médicos, enfermeiros, fisioterapeutas, terapeutas ocupacionais, assistentes sociais, psicólogos, nutricionistas ${ }^{(19)}$ e as intervenções devem ser adaptadas à fase de doença, à pessoa em causa e à sua capacidade de aprendizagem, ao local onde são realizadas e aos meios disponíveis ${ }^{(6)}$.

Esta revisão da literatura evidenciou que a reabilitação respiratória foi realizada por vários profissionais de saúde, enfermeiros, fisioterapeutas, terapeutas, técnicos e médicos ${ }^{(11,12,15,16)}$.

Os sintomas mais frequentes das patologias respiratórias, que levam as pessoas a procurar cuidados de saúde foram a dispneia, a tosse e a intolerância ao exercício ${ }^{(6)}$. A presença de dispneia, a intolerância ao exercício e a limitação nas atividades da vida diária, levam a uma perda progressiva da capacidade funcional e à adoção de estilos de vida sedentários e são estes critérios que estão normalmente presentes na decisão para implementação de um programa de reabilitação respiratória ${ }^{(20)}$.

Nos artigos analisados, para além destes sinais ou sintomas, outros, como a tosse ineficaz, a intolerância ao esforço(11), a fraqueza muscular dos músculos respiratórios e dos músculos que intervêm na deglutição ${ }^{(12,15)}$, a diminuição dos volumes pulmonares ${ }^{(13,14)}$, o aumento da fadiga ${ }^{(15)}$ e a diminuição da capacidade vital em geral ${ }^{(17)}$ foram as manifestações que determinaram a necessidade de intervenção de reabilitação respiratória.

As pessoas com doença pulmonar obstrutiva crónica (DPOC), foram na maioria dos casos os alvos principais destas terapias ${ }^{(18)}$. A análise dos estudos que incluíram esta revisão, para além dos benefícios nos doentes com $\operatorname{DPOC}^{(11,13,16)}$ identificou também benefícios em pessoas com outras patologias como o acidente vascular cerebral $(\mathrm{AVC})^{(12)}$, a doença de Parkinson ${ }^{(14)}$ a miastenia gravis $^{(15)}$, a obesidade ${ }^{(16)}$ e a asma ${ }^{(17)}$, situações que cursam com patologias respiratórias crónicas ou que apresentam risco de desenvolver uma perda progressiva da capacidade funcional levando a pessoa a adotar estilos de vida sedentários promotores de isolamento social e que por isso são suscetíveis de beneficiar de reabilitação respiratória ${ }^{(20)}$.

Quando nos referimos à reabilitação respiratória estamos a englobar várias intervenções integrantes como a reeducação funcional respiratória, o treino de tolerância ao exercício, a otimização da terapia inalatória e a assistência respiratória ${ }^{(5)}$. Como as intervenções são personalizadas segundo as necessidades de cada doente, nem todos os elementos da reabilitação respiratória precisam de ser aplicados ${ }^{(18)}$.

A reeducação funcional respiratória baseia-se na associação do controlo da respiração, do posicionamento e do movimento, ou seja, no restabelecimento funcional da respiração ${ }^{(21)}$. De acordo com um dos estudos, em doentes com DPOC e dispneia moderada as intervenções relacionadas com estes exercícios tiveram como resultados nos doentes a melhoria dos volumes pulmonares e melhoria da ventilação ${ }^{(13)}$

Noutros doentes com DPOC, a consciencialização da doença ${ }^{(13)}$, as técnicas de limpeza de vias aéreas, a técnica de respiração com lábios semicerrados, os exercícios de membros superiores com inspiração profunda, o treino de marcha e a educação para a saúde tiveram como benefícios para os doentes a diminuição da dispneia, a diminuição da frequência de tosse e maior tolerância ao exercício ${ }^{(11,13)}$.

A dispneia é uma das causas que levam as pessoas com problemas respiratórios a procurar cuidados de saúde com maior frequência. Nos estudos analisados, a técnica de controlo da dispneia mais utilizada foi a respiração com os lábios semicerrados ${ }^{(22)}$. Esta técnica, pode ser incluída durante a realização das atividades da vida diária e exercícios como caminhadas ${ }^{(23)}$. Ao permitir o aumento do volume corrente e o controlo do padrão respiratório, esta técnica permite aumentar o tempo expiratório, esvaziando de forma mais eficaz o ar acumulado nos pulmões ${ }^{(24)}$. No estudo de Ubolnuar e colaboradores (2020), o uso desta técnica permitiu aumentar o volume corrente e o aumento do fluxo inspiratório e expiratório médio em doentes com DPOC ${ }^{(13)}$.

Com o avançar da idade, mas também por disfunção neurológica, o reflexo de tosse pode ficar mais deprimido e os doentes ficam mais suscetíveis a infeção respiratória ${ }^{(3)}$. Os músculos inspiratórios e expiratórios que funcionam como os outros músculos esqueléticos tendem a diminuir a força, o que também pode acontecer por malnutrição e com a imobilidade ${ }^{(3)}$. 
Os músculos expiratórios são recrutados para manter níveis elevados de ventilação durante o exercício de manobras expiratórias forçadas, como no treino da tosse ${ }^{(6)}$. Adicionalmente o treino de músculos inspiratórios através do uso de dispositivos, na medida em que estes permitem o uso de pressões mais elevadas que causam resistência à inspiração acabam por permitir posteriormente pressões normais durante a expiração, cujo resultado é uma expiração mais próxima do normal e mais relaxada ${ }^{(6)}$. Este tipo de benefícios também foi conseguido em doentes com AVC associando ao treino dos músculos o treino postural e a técnica da tosse com melhorias na capacidade vital e na fadiga destes doentes ${ }^{(12)}$.

Em doentes com miastenia gravis, o treino de fortalecimento de músculos inspiratórios e expiratórios pelo uso de pressões durante três meses também demonstrou ser eficaz na redução da fadiga física e consequente melhoria funcional ${ }^{(15)}$. Em doentes com asma, verificou-se um aumento da capacidade de marcha durante seis minutos ${ }^{(17)}$.

Embora alguns autores reconheçam os benefícios do uso de dispositivo para treino dos músculos inspiratórios ${ }^{(15,17)}$, outros defendem que o uso destes dispositivos ainda que melhore a fadiga e os volumes pulmonares, é uma técnica viável como terapia adjuvante, mas que deve ser complementada com outras técnicas de reabilitação respiratória, para melhores resultados ${ }^{(12)}$.

Um dos objetivos da reabilitação respiratória, prende-se com a eliminação de secreções e a manutenção da permeabilidade das vias aéreas, quando há problemas obstrutivos ${ }^{(25)}$. A limpeza das vias aéreas engloba não só a expulsão, mas também a mobilização das secreções brônquicas ${ }^{(4)}$, isto é, técnicas de limpeza de vias aéreas.

O programa de reabilitação de Liao e colaboradores (2015) proporcionou aos doentes com DPOC ${ }^{(11)}$ ensinos sobre técnicas de limpeza de vias aéreas, como a drenagem postural, tanto ao doente como familiar, de forma a permitir uma assistência adequada.

Tal como já foi referido, a dispneia é uma das causas mais frequentes que levam as pessoas a procurar os cuidados de saúde. A técnica de controlo da dispneia mais utilizada é a estratégia de respiração com os lábios semicerrados ${ }^{(22)}$. Esta técnica permite aumento do volume corrente, controlo do padrão respiratório e o esvaziamento máximo dos alvéolos, uma vez que os elementos de menor calibre da via aérea inferior, permanecem abertos por períodos mais prolongados durante o tempo expiratório, permitindo assim aumentar o tempo expiratório e esvaziando de forma mais eficaz o ar acumulado nos pulmões ${ }^{(24)}$.

Ubolnuar e colaboradores $(2020)^{(13)}$ demonstram que o uso da técnica de respiração com os lábios semicerrados permite um aumento do tempo inspiratório e expiratório. $O$ uso desta técnica em conjugação com outras, como a coordenação com a respiração abdominodiafragmática aumentam a eficiência do diafragma e diminuem a assincronia toracoabdominal verificada durante os períodos de dispneia, tendo como resultado a sua melhoria ${ }^{(3,}$ 11,16).

A conjugação da técnica de controlo respiratório com a técnica de abertura costal global também permite a redução de dispneia e aumento de tolerância ao exercício proposto ${ }^{(11,16)}$.

Ribeiro e colaboradores $(2018)^{(14)}$ fazem menção às técnicas de inspirometria de incentivo e breath staking para promoção de expansão pulmonar. Esta última tem como objetivo a expansão pulmonar através da utilização de uma máscara facial que envolve o nariz e a boca e é conectada a uma válvula unidirecional, que permite a entrada do ar inspirado e bloqueia o ar expirado, sendo as pessoas instruídas a realizarem inspirações a partir da capacidade residual funcional até à capacidade pulmonar total ${ }^{(26)}$. Esta técnica permite melhorar a função respiratória com aumento do volume pulmonar e normalização da frequência respiratória ${ }^{(26)}$.

A inspirometria de incentivo frequentemente usada nos períodos pré e pós cirúrgico, com a finalidade de diminuir o risco de complicações respiratórias, associadas à diminuição do volume pulmonar após a cirurgia ${ }^{(27)}$, também foi utilizada em doentes com patologias do foro neurológico ${ }^{(5,14)}$, com efeitos benéficos nos seus volumes correntes ${ }^{(14)}$. Ficou demonstrado também que os resultados são semelhantes entre as duas técnicas (inspirometria de incentivo e breath staking) e a escolha é influenciada de acordo com o estado de consciência e cooperação dos doentes para a realização destas técnicas, tendo em conta que a inspirometria de incentivo exige maior colaboração da pessoa ${ }^{(14)}$.

\section{CONCLUSÃO}

Os estudos analisados permitem concluir que as técnicas de reabilitação respiratória usadas como forma de tratamento não farmacológico para controlo dos sintomas têm como resultados nos doentes adultos e idosos a redução da fadiga e diminuição da dispneia, o aumento da tolerância ao esforço, as alterações nos volumes e capacidades pulmonares, bem como alterações nas pressões inspiratória e expiratória máximas.

Não foram apenas as pessoas com patologia respiratória que beneficiaram das intervenções de reabilitação respiratória, uma vez que os mais idosos ou as pessoas com patologias do foro neurológico também obtiveram resultados na melhoria no desempenho das suas atividades da vida diária.

Como limitações para a pesquisa, podemos referir o baixo número de artigos enquadrados na temática desenvolvida e o facto de a mesma ter sido conduzida apenas na plataforma EBSCOhost. Sugerimos a realização de mais estudos 
de verificação da eficácia das diferentes intervenções de forma que a prática de reabilitação respiratória seja conduzida de acordo com a melhor evidência científica.

\section{REFERÊNCIAS BILIOGRÁFICAS}

1. Radwanski, M. L. Enfermagem de Reabilitação Gerontológica. IN Hoeman, S.P. Enfermagem de Reabilitação- Prevenção, Intervenção e Resultados esperados. Loures: Lusodidacta, 2011: $4^{\mathrm{a}}$ edição, pp.695-714.

2. Diário da República $n .^{\circ}$ 85/2019, Série II de 2019-05-03. Regulamento $n .^{\circ}$ 392/2019. Regulamento das competências específicas do enfermeiro especialista em Enfermagem de Reabilitação. Ordem dos Enfermeiros. Disponível em: https://dre.pt/web/guest/pesquisa//search/122216893/details/normal?l=1

3. Branco, P. S.; Barata, S.; Barbosa, J.; Cantista, M.; Lima, A.; Maia, J. Temas de Reabilitação- Reabilitação Respiratória. Porto: Medesign.[Internet] 2012 [citado 2021 Jun 27] Disponível em: https://docplayer.com.br/6449699-Temas-de-reabilitacao-reabilitacaorespiratoria-pedro-soares-branco-e-colaboradores.html

4. Global Initiative for Chronic Obstructive Lung Disease. GLOBAL STRATEGY FOR THE DIAGNOSIS, MANAGEMENT, AND PREVENTION OF CHRONIC OBSTRUCTIVE PULMONARY DISEASE. [Internet] 2006 [citado 2021 julho 5]. Disponível em: https://www.who.int/respiratory/copd/GOLD_WR_06.pdf

5. Cordeiro, M. C. O; Menoita, E. C. P. C. Manual de boas práticas na reabilitação respiratória: Conceitos, Princípios e Técnicas. Lusociência: 2012: $1^{\mathrm{a} e d}$

6. Gronkiewicz, C; Coover, L. Reabilitação respiratória e pulmonar. [autor do livro] Hoeman, S.P. Enfermagem de Reabilitação- Prevenção, Intervenção e Resultados esperados. Loures: Lusodidacta, 2011: $4^{a}$ edição: pp.319-350

7. Institute TJB. Joanna Briggs Institute Reviewers' Manual: 2014 edition [Internet]. Adelaide: The Joanna Briggs Institute; 2014. 3-195 p. Online:

8. https://docplayer.net/6678504-Joanna-briggs-institute-reviewers-manual-2014-edition.html

9. Institute TJB. The Joanna Briggs Institute Reviewers' Manual 2015. [Internet] The Joanna Briggs Institute, 2015. Edition 1, pp.1-24. Online: https://www.researchgate.net/publication/294736492_Methodology_for_JBI_Scoping_Reviews

10. Institute TJB. JBI Levels of Evidence. JBI approach [Internet]. 2013. Outubro:2-6. Online: https://joannabriggs.org/sites/default/files/201905/JBI-Levels-of-evidence_2014_0.pdf

11. Institute TJB. JBI Approach : Grades of Recommendation. [Internet] $2013 . \quad$ Outubro. Online: https://joannabriggs.org/sites/default/files/2019-05/JBI-grades-of-recommendation_2014.pdf

12. Liao, L.-Y., Chen, K.-M., Chung, W.-S., \& Chien, J.-Y. (2015). Efficacy of a respiratory rehabilitation exercise training package in hospitalized elderly patients with acute exacerbation of COPD: a randomized control trial. International Journal of Chronic Obstructive Pulmonary Disease [Internet] 2015 [citado 2021 Jun 27] 1703-1709. Disponível em: Doi:10.2147/copd.s90673

13. Liaw, M.-Y., Hsu, C.-H., Leong, C.-P., Liao, C.-Y., Wang, L.-Y., Lu, C.-H., \& Lin, M.-C. Respiratory muscle training in stroke patients with respiratory muscle weakness, dysphagia, and dysarthria - a prospective randomized trial. Medicine. [Internet]. 2020. [citado 2021 Jun 27] 99(10).Disponível em: Doi:10.1097/md.0000000000019337

14. Ubolnuar,N; Tantisuwat, A; Thaveeratitham,P; Lertmaharit,S; Kruapanich,C; Chimpalee, J; Mathiyakom, W. Effects of pursed-lip breathing and forward trunk lean postures on total and compartmental lung volumes and ventilation in patients with mild to moderate chronic obstructive pulmonary diseas. An observational study. Medicine [Internet] Dezembro 2020. [ citado 2021 Jun 2021] 18; 99(51). Disponível em Doi: 10.1097/MD.0000000000023646

15. Ribeiro, R., Brandão, D., Noronha, J., Lima, C., Fregonezi, G., Resqueti, V., \& Dornelas de Andrade, A. Breath-stacking and incentive spirometry in Parkinson's disease: Randomized crossover clinical trial. Respiratory Physiology \& Neurobiology. [Internet] 2018. [citado 2021 Jun 27] Disponível em Doi:10.1016/j.resp.2018.04.011

16. Hsu, C.-W., Lin, H.-C., Tsai, W.-C., Lai, Y.-R., Huang, C.-C., Su, Y.-J., Lu, C-H, Lin, W-C; Chang, C-L; Chang, W-N; Lin M-C; Tsai, N.W. Respiratory Muscle Training Improves Functional Outcomes and Reduces Fatigue in Patients with Myasthenia Gravis: A Single-Center Hospital-Based Prospective Study. BioMed Research International [Internet] 2020. [citado 2021 Jun 27] pp 1-8. Disponível em Doi:10.1155/2020/2923907

17. Martinelli, B., Pires Di Lorenzo, V. A., Quitério, R. J., Ambrozin, A. R. P., Arca, E. A., \& Jamami, M. Cardiorespiratory repercussions according to the abdominal circumference measurement of men with obstructive respiratory disorder submitted to respiratory physiotherapy. Physiotherapy Theory and Practice [Internet] 2018 [citado 2021 Jun 27] 34(11), 835-845. Disponível em doi:10.1080/09593985.2018.1430195

18. Chung, Y.; Huang, T.-Y.; Liao, Y.-H.; Kuo, Y.-C. 12-Week Inspiratory Muscle Training Improves Respiratory Muscle Strength in Adult Patients with Stable Asthma: A Randomized Controlled Trial. International Journal of Environmental Research and Public Health. [Internet] 2021 [citado 2021 Jun 27] 18, 3267. Disponível em: https://doi.org/10.3390/ijerph18063267

19. Heitor, M. C. Canteiro, M. C.(2003). Reabilitação Respiratória. [autor do livro] Gomes, M. J. M.; Sotto- Mayor, R. Tratado de Pneumologia. Sociedade Portuguesa de Pneumologia: 2003, Vol. I: pp.1785-1787.

20. Spruit, M. A., Singh, S. J., Garvey, C., ZuWallack, R., Nici, L., Hill, K.; Holland, A. E.; Lareau,S. C.; Man, W. D-C.; Pitta, F.; Sewell, L.; Raskin, J.; Bourbeau, Crouch, R.; Framssem, F. M. E.; Casaburi, R.; Vercoulen, Vogiatzis, J.; Gosselink, R.; ... Wouters, E. F. M. An Official American Thoracic Society/European Respiratory Society Statement: Key Concepts and Advances in Pulmonary Rehabilitation. American Journal of Respiratory and Critical Care Medicine [Internet] 2013 [citado 2021 jun 27] 188(8), e13-e64. Disponível em: doi:10.1164/rccm.201309-1634st

21. Bárbara, C; Canteiro, M. C. Selecção, avaliação e monitorização do doente candidato a um Programa de Reabilitação Respiratória. [autor do livro] Gomes, M. J. M.; Sotto- Mayor, R. Tratado de Pneumologia. Sociedade Portuguesa de Pneumologia: 2003, Vol. I: pp.1789-1794.

22. Canteiro,M. C.; Heitor, M. C. Olazabal, M.; Abreu, P. J. Reeducação funcional respiratória. [autor do livro] Gomes, M. J. M.; Sotto- Mayor, R. Tratado de Pneumologia. Sociedade Portuguesa de Pneumologia: 2003, Vol. I: pp.1806-1812.

23. Direcção Geral da Saúde (DGS). Orientações técnicas sobre reabilitação respiratória na doença pulmonar obstrutiva crónica (DPOC). Ministério da Saúde. [Internet] 2009. [citado 2021 Jun 27] Disponível em: https://www.dgs.pt/directrizes-da-dgs/orientacoes-e-circularesinformativas/circular-informativa-n-40adspcd-de-27102009-pdf.aspx

24. Nield, M.A.; Soo Hoo, G.W.; Roper, J. M.; Santiago, S. Efficacy of Pursed-Lips Breathing-A breathing pattern retraining strategy for dyspnea reduction. Journal of Cardiopulmonary Rehabilitation and Prevention [Internet] 2007 [citado 2021 Jun 27] 27:237/244. Disponível em: doi: 10.1097/01.HCR.0000281770.82652.cb

25. Larson, J. L.; Johnson, J. H.; Angst, D. B. Função Respiratória e Reabilitação Pulmonar. [autor do livro] Hoeman, S. P. Enfermagem de 
Reabilitação- Aplicação e Processo. Lusociência: 2000, $2^{\circ}$ Edição: pp. 393- 433.

26. Olazabal, M. Métodos de limpeza das vias aéreas. [autor do livro] Gomes, M. J. M.; Sotto- Mayor, R. Tratado de Pneumologia. Sociedade Portuguesa de Pneumologia: 2003, Vol. I: pp.1807-1812.

27. Vaz, S. F. A.; Matos, T. F. V; Mendes, M. E. R; Preto, L. S. R; Fernandes, H. J; Novo, A. F. M. P. Eficácia da técnica de breath stacking na função respiratória em mulheres submetidas a cirurgia bariátrica. Revista de Enfermagem Referência [Internet] 2019 [citado 2021 Jun 27] SérielV,23(pp.49-58) Disponível em: https://doi.org/10.12707/RIV19046.

28. Nascimento Junior, P. Módolo, N. S. P.; Andrade, S.; Guimarães, M. M. F.; Braz,L. G.; Dib, R. Incentive spirometry for prevention of postoperative pulmonary complications in upper abdominal surgery.Cochrane Database Of Systematic Reviews.[Internet] 2014 [citado 2021 Jun 27] (2). Disponível em: doi:10.1002/14651858.CD006058.pub3.

\section{DIVULGAÇÕES ÉTICAS}

Contribuição do(s) autor(es):

Conceptualização: JÁ; EG;

Metodologia: JÁ; EG;

Validação: JÁ; EG;

Análise formal: JÁ; EG;

Investigação: JÁ; EG;

Tratamento de dados: JÁ; EG;

Preparação do rascunho original: JÁ; EG;

Redação e edição: JÁ; EG;

Revisão: JÁ; EG;

Todos os autores leram e concordaram com a versão publicada do manuscrito.

Financiamento:

Este trabalho não recebeu nenhuma contribuição financeira ou bolsa.

Comissão de Ética:

Não se aplica.

Declaração de consentimento informado:

Não se aplica.

Conflitos de interesse:

Os autores não declaram nenhum conflito de interesses.

Proveniência e revisão por pares:

Não comissionado; revisto externamente por pares.

(c) Autor (es) (ou seu (s) empregador (es)) e APER/RPER 2022. Reutilização permitida de acordo com CC BY-NC-ND. Nenhuma reutilização comercial. 